\title{
Relación entre hipoacusia y diabetes mellitus tipo 2
}

\author{
Relationship between hearing loss and type 2 diabetes mellitus
}

Cesar Imarai $\mathbf{B}^{1}$, Karina Aracena ${ }^{2}$, Daniela Contreras $\mathbf{M}^{1}$, Jorge Caro $\mathbf{L}^{1}$.

\begin{abstract}
RESUMEN
Introducción: La diabetes mellitus tipo 2 es una enfermedad prevalente en nuestro país. Básicamente en esta, falla la regulación de la glicemia lo que puede provocar diversas complicaciones. Diversos estudios han encontrado que puede causar lesiones a largo plazo en el sistema auditivo pudiendo llegar a causar una hipoacusia de tipo neurosensorial.

objetivo: Conocer si pacientes con diabetes mellitus tipo 2 presentan una hipoacusia sensorio neural mayor de lo esperado.

Material y método: Estudio transversal caso control. El grupo comparativo estuvo constituido por 45 pacientes con diabetes mellitus tipo 2 y con 53 pacientes sanos y que constituyen el grupo control. La edad de ambos grupos fluctuó entre 30 a 50 años. Los criterios de exclusión incluyeron a pacientes con trauma acústico, ototóxicos y antecedentes otológicos previos. Además se aplicó una encuesta y un examen otológico para descartar lesiones. A cada paciente se le realizó una audiometría tonal, siempre en las mismas condiciones y por el mismo examinador. Se analizaron en cada oído las frecuencias 128; 256; 512; 1.000; 2.000; 3.000; 4.000; 6.000; $8.000 \mathrm{~Hz}$ y el PTP y en intervalos de $5 \mathrm{~dB}$ en cada frecuencia. Para el análisis estadístico, se aplicó las pruebas de normalidad de Kolmogorov-Smirnov y Shapiro-Wilk y la prueba no paramétrica de Mann Whitney.

Resultados: La media de las edades fue de 40,85 años con desviación estándar 6,33 para los controles y 42,62 años con desviación estándar de 5,97 para el grupo de diabéticos. El tiempo de evolución promedio de diabetes fue de 5,03 años. En el análisis de la diferencia en los promedios tonales se observó un deterioro significativo $(P<0,05)$ en el grupo de diabéticos con respecto a los controles en casi todas las frecuencias evaluados y también en el PTP en ambos oídos. La excepción a lo anterior solo se observó en la frecuencia $256 \mathrm{~Hz}$ del oído derecho.

Conclusiones: Los pacientes con diabetes mellitus tipo 2 de nuestro estudio presentaron una pérdida auditiva tonal estadísticamente significativa al compararlos con el grupo control.

Palabras clave: Diabetes mellitus tipo 2, hipoacusia sensorio neural, audiometría tonal.

ABSTRACT

Introduction: Diabetes mellitus is a prevalent disease in Chile, in diabetes glucose disregulation can cause organ and system failure. Several studies have found that diabetes can also cause long-term injuries in the auditory system, causing neurosensorial hearing loss.

Aim: To associate diabetes mellitus and the potential consequence of hearing loss determined by tonal audiometry.
\end{abstract}

\footnotetext{
${ }^{1}$ Médico del Servicio de Otorrinolaringología, Hospital Clínico de la Pontificia Universidad Católica de Chile.

2 Tecnóloga Médico del Servicio de Otorrinolaringología, Hospital Clínico de la Pontificia Universidad Católica de Chile.
} 
Material and method: Cross-sectional study case-control, with a $N=45$ for diabetic patients and $N=53$ for control subjects, between 30 to 50 years old. We excluded patients with acoustic trauma, ototoxic use or previous otologic history. A questionnaire was applied and otologic examination was done to rule out injuries. Audiometry was performed, analyzing frequencies in each ear 128, 256, 512, 1000,2000,3000,4000,6000,8000 Hz and PTP. For statistical analysis we applied the nonparametric Mann Whitney test.

Results: Mean age was 40.85 years old with standard deviation 6.33 for controls and 42.62 years with standard deviation of 5.97 for cases, average duration of diabetes was of 5.03 years. In the analysis of the difference in average pitch there was a higher significant deterioration $(P<0.05)$ in the diabetic group compared to controls in almost all frequency and PTP in both ears, except in the $256 \mathrm{~Hz}$ right ear.

Conclusions: Patients with diabetes mellitus showed a hearing loss in tonal audiometry compared to individuals without diabetes mellitus.

Key words: Diabetes, neurosensorial hearing loss, tonal audiometry.

\section{INTRODUCCIÓN}

La diabetes mellitus tipo 2 (DM) es una enfermedad prevalente en nuestro país, siendo provocada por una falla en los mecanismos de regulación de la glicemia, generando hiperglicemias crónicas, que a lo largo del tiempo provocan diversas complicaciones en órganos blancos como la retina, riñón y extremidades ${ }^{1}$. Las complicaciones son el resultado de lesiones a nivel endotelial, neural y matriz extracelular de los tejidos. Todas ellas finalmente llevan a un gran estrés oxidativo².

Según la encuesta nacional de salud la prevaIencia de esta enfermedad fue de $6,3 \%$ en 2003 , subiendo a $9,4 \%$ en la encuesta de $2009-2010^{3}$. Epidemiológicamente Chile es uno de los países en América con las mayores tasas de diabetes mellitus tipo 2, con prevalencia e incidencia crecientes ${ }^{4}$.

El diagnóstico de diabetes mellitus tipo 2 en niños y adolescentes es cada vez más frecuente ${ }^{1}$, y esto seguramente producirá un aumento de complicaciones crónicas por esta enfermedad en pacientes más jóvenes.

Por más de 100 años, diversos estudios han tratado de correlacionar la diabetes y la pérdida auditiva con resultados controversiales. Algunos estudios han encontrado que la DM tipo 2 puede causar lesiones en el sistema auditivo en el largo plazo ${ }^{5}$. La lesión es una hipoacusia de tipo neurosensorial. Esta sería mayor a la esperada con respecto a la edad en sujetos normales y sin antecedentes auditivos. La severidad de la pérdida auditiva dependería también de lo avanzado de la enfermedad metabólica ${ }^{6}$.
La diabetes mellitus podría causar posibles daños a nervios y vasos del oído interno, que podrían llevar a una degeneración neuronal del aparato auditivo ${ }^{7}$. Los estudios en modelos animales han encontrado engrosamiento de la membrana basal, en ratones con diabetes inducida, los que son concordantes con lesiones de microangiopatía ${ }^{8}$.

Hyun-Seok y col en un modelo experimental con ratones, demostró lesiones en el órgano de Corti, de células del ganglio espiral, y también degeneración coclear temprana en ratones obesos con hiperglicemias severas ${ }^{9}$.

En estudios en hueso temporal de diabéticos tipo 2, se encontraron lesiones en el saco endolinfático, en la membrana basilar y lesiones de microangiopatía en la estría vascular, que se relacionaron con hipoacusia pero ellas fueron sin significado estadístico ${ }^{10}$.

Fukushima y col también demostraron lesiones en estría vascular y en células ciliadas externas en 18 huesos temporales de pacientes diabéticos, que se trataban con hipoglicemiantes o insulina, observados bajo microscopio electrónico ${ }^{11}$.

Cullen JR demostró que pacientes diabéticos insulino dependientes eran significativamente más hipoacúsicos que el grupo control ${ }^{12}$. Díaz de León Morales y col encuentran pérdidas auditivas subclínicas en frecuencias agudas y deterioro de la respuesta auditiva del tronco cerebral, pero ésta fue independiente de otras complicaciones de microangiopatía, como retinopatía, neuropatía o nefropatía diabética ${ }^{13}$.

Sin embargo hay otros autores, como De España R. que concluye en su trabajo que la pérdida 
auditiva no es una característica habitual de los pacientes con DM, y que cuando se presenta puede ser atribuida a deterioro fisiológico del oído interno por envejecimiento y que los mecanismos patogénicos son oscuros ${ }^{14}$.

Para este trabajo nuestra hipótesis diagnóstica fue: los pacientes con diabetes mellitus tienen una mayor pérdida del umbral auditivo que sujetos normales sin esta patología.

\section{OBJETIVO}

El objetivo de este trabajo fue conocer si pacientes con diabetes mellitus tipo 2 presentan una hipoacusia sensorioneural mayor de lo esperado. Así se planteó un estudio de asociación entre la diabetes mellitus y la pérdida auditiva mediante audiometría tonal.

\section{MATERIAL Y MÉTODO}

Este estudo fue de tipo prospectivo transversal caso control, comparando dos grupos de estudio. Un grupo de 45 pacientes diabéticos tipo 2 elegidos al azar y un grupo de 53 controles sanos y que cumplieran con los criterios de exclusión. Para ambos grupos con antecedentes de trauma acústico, uso de ototóxicos, malformaciones y antecedentes de hipoacusia previa. En ambos grupos las edades fluctuaron entre 30 y 50 años. La elección del grupo control también fue al azar y se intentó ser lo más semejante al grupo de diabéticos.

En el grupo de diabéticos, 44 pacientes estaban con tratamiento dietético y la mayoría de ellos con hipoglucemiantes orales durante varios años. Sólo un paciente era usuario recientemente de insulina por mal control metabólico.

Se aplicó un consentimiento informado aprobado por el Comité de Ética de la Universidad Católica de Chile y una escuesta a los individuos estudiados (diabéticos y controles), donde se recopilaron los datos de sexo y edad, tiempo de evolución de diabetes y tratamiento si correspondía, antecedentes mórbidos, enfermedades otológicas previas, exposición a trauma acústico y exposición a ototóxicos.

Luego se realizó un examen otológico mediante otoscopio u otomicroscopio para descartar lesiones que fueran causa de hipoacusia y se realizó una audiometría tonal, con audiómetro MADSEN 622, que fue realizado siempre en el mismo lugar y por el mismo tecnólogo médico. Se analizó en cada oído el umbral de audición en las frecuencias 128, 256, $512,1.000,2.000,3.000,4.000,6.000,8.000 \mathrm{~Hz}$ y el promedio tonal puro (PTP) en intervalos de $5 \mathrm{~dB}$.

No se evaluó la discriminación ni la variación individual auditiva en ambos grupos estudiados.

Los datos fueron analizados por el Departamento de Estadísticas de Centro de Investigaciones Médicas de la Universidad Católica de Chile.

Para el análisis estadístico, se aplicó las pruebas de normalidad de Kolmogorov-Smirnov y ShapiroWilk y la prueba no paramétrica de Mann Whitney, para el análisis de las diferencias de rangos promedios de los umbrales auditivos en las distintas frecuencias de ambos grupos, que son comparables, considerando un valor de $p<0,05$ como significativo.

\section{RESULTADOS}

Las medias de las edades fueron de 40,85 años con desviación estándar de 6,33 para el grupo de control, mientras que para el grupo de diabetes fue de 42,62 años con desviación estándar de 5,97 (Tabla 1).

Tabla 1. Promedio de edad de grupo diabetes y control

\begin{tabular}{|c|c|c|c|}
\hline Grupo & $\begin{array}{c}\text { Edad } \\
\text { media }\end{array}$ & DS & $\begin{array}{c}\text { Rango } \\
\text { edad }\end{array}$ \\
\hline Diabético & 42,62 & 5,97 & $30-50$ \\
\hline Control & 40,85 & 6,33 & $30-50$ \\
\hline
\end{tabular}

En cuanto al género, el grupo control contó con $58,5 \%$ de mujeres y $41,5 \%$ de hombres, en el grupo de diabetes contó con $64,4 \%$ de mujeres y $35,6 \%$ de hombres (Tabla 2). El tiempo de evolución promedio diabetes mellitus fue de 5,03 años.

Tabla 2. Distribución por género de grupo de diabetes y control

\begin{tabular}{|c|c|c|}
\hline Grupo & Femenino & Masculino \\
\hline Diabético & $64,4 \%$ & $35,6 \%$ \\
\hline Control & $58,5 \%$ & $41,5 \%$ \\
\hline
\end{tabular}




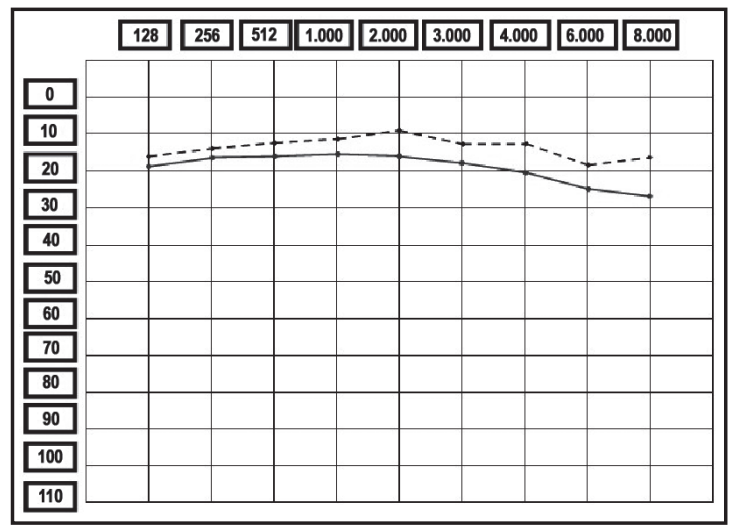

Figura 1. Media de umbrales auditivos oído izquierdo, línea continua corresponde a pacientes diabéticos y línea discontinua corresponde a pacientes controles.

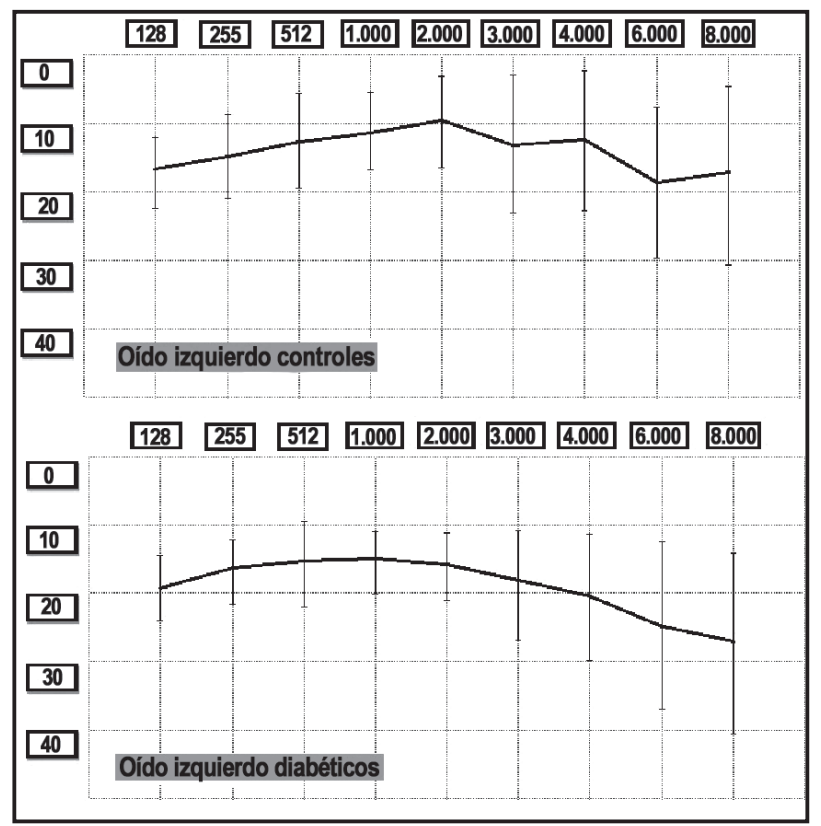

Figura 2. Umbrales auditivos oído izquierdo de pacientes controles y diabéticos con desviación típica.

Tabla 3. Media de umbrales auditivos del oído izquierdo de controles y diabéticos.

\begin{tabular}{|c|c|c|c|c|c|}
\hline Frecuencia & $\begin{array}{l}\text { Media dB } \\
\text { Diabéticos }\end{array}$ & $\begin{array}{c}\text { Desviación } \\
\text { Típica }\end{array}$ & $\begin{array}{l}\text { Media dB } \\
\text { Controles }\end{array}$ & $\begin{array}{c}\text { Desviación } \\
\text { Típica }\end{array}$ & $\mathrm{P}$ \\
\hline 128 & 19,56 & 3,9 & 16,79 & 5,8 & 0,002 \\
\hline 256 & 16,22 & 4,9 & 14,43 & 6 & 0,073 \\
\hline 512 & 17,11 & 5,1 & 12,45 & 6,1 & $<0,001$ \\
\hline 1000 & 14,78 & 3,5 & 11,89 & 6 & 40,001 \\
\hline 2000 & 13,78 & 5 & 9,43 & 6,6 & $<0,001$ \\
\hline 3000 & 17,98 & 8,8 & 11,7 & 9,2 & $<0,001$ \\
\hline 4000 & 19,33 & 10 & 14,83 & 11,4 & 0,003 \\
\hline 6000 & 26,78 & 12 & 19,72 & 11,9 & 0,002 \\
\hline 8000 & 24,11 & 11,5 & 16,89 & 14,6 & $<0,001$ \\
\hline
\end{tabular}

Tabla 4. Media de umbrales auditivos del oído derecho de controles y diabéticos.

\begin{tabular}{|c|c|c|c|c|c|}
\hline Frecuencia & $\begin{array}{l}\text { Media dB } \\
\text { Diabéticos }\end{array}$ & $\begin{array}{c}\text { Desviación } \\
\text { Típica }\end{array}$ & $\begin{array}{l}\text { Media dB } \\
\text { Controles }\end{array}$ & $\begin{array}{c}\text { Desviación } \\
\text { Típica }\end{array}$ & $\mathrm{P}$ \\
\hline 128 & 19,56 & 3,9 & 16,79 & 5,8 & 0,002 \\
\hline 256 & 16,22 & 4,9 & 14,43 & 6 & 0,073 \\
\hline 512 & 17,11 & 5,1 & 12,45 & 6,1 & $<0,001$ \\
\hline 1000 & 14,78 & 3,5 & 11,89 & 6 & $<0,001$ \\
\hline 2000 & 13,78 & 5 & 9,43 & 6,6 & $<0,001$ \\
\hline 3000 & 17,98 & 8,8 & 11,7 & 9,2 & $<0,001$ \\
\hline 4000 & 19,33 & 10 & 14,83 & 11,4 & 0,003 \\
\hline 6000 & 26,78 & 12 & 19,72 & 11,9 & 0,002 \\
\hline 8000 & 24,11 & 11,5 & 16,89 & 14,6 & $<0,001$ \\
\hline
\end{tabular}


En el análisis de la diferencia de las medias de intensidad de umbrales auditivos, se observó un deterioro en el grupo de diabéticos con respecto a los controles, que fue estadísticamente significativo $(p<0,05)$ en todas las frecuencias audiométricas de cada oído, salvo en la frecuencia $256 \mathrm{~Hz}$ del oído derecho ( $p=0,073$ ).

En las Figuras 1, 2 y Tabla 3 se observa una diferencia significativa en todas las frecuencias auditivas del oído izquierdo, siendo peor las medias de intensidad de

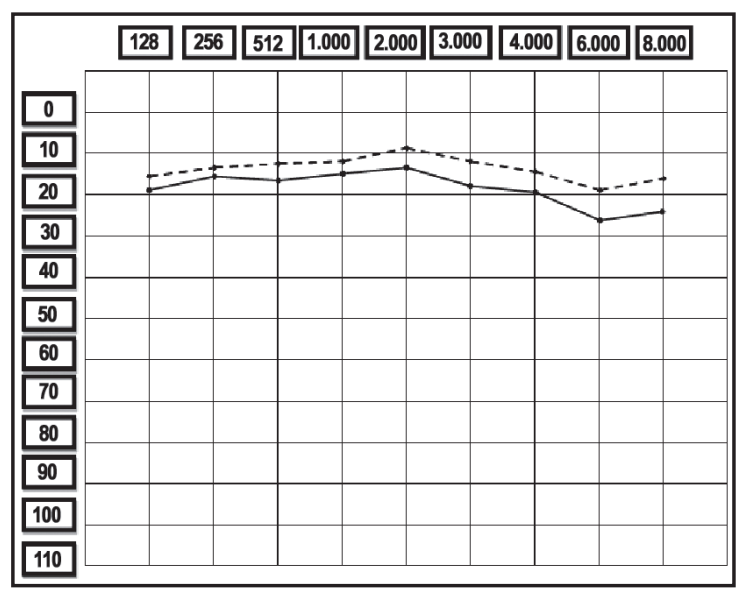

Figura 3. Media de umbrales auditivos oído derecho, línea continua corresponde a pacientes diabéticos y línea discontinua corresponde a pacientes controles. umbrales medidas en los pacientes portadores de diabetes mellitus. Este deterioro es más marcado en las frecuencias medias y agudas.

En las Figura 3, 4 y Tabla 4, que muestra las mediciones en el oído derecho, también encontramos un diferencia significativa en la media de las intensidades de umbrales auditivos, siendo peor en el grupo de pacientes diabéticos, aunque en la frecuencia audiométrica $256 \mathrm{~Hz}$, si bien hay un deterioro no fue significativo.

La Figura 5, es un promedio de la media de intensidad de umbral por cada frecuencia de ambos oídos, para demostrar el panorama general de los resultados.

El promedio tonal puro también tuvo una diferencia significativa en ambos oídos, encontrando mayor pérdida en el grupo de diabéticos (Tabla 5). En ambos grupos la discriminación con disílabos fue normal.

\section{DISCUSIÓN}

Nuestra población tiene una de las tasas de diabéticos más altas en América. La prevalencia de la DM tipo 2 ha ido en aumento en la última década.

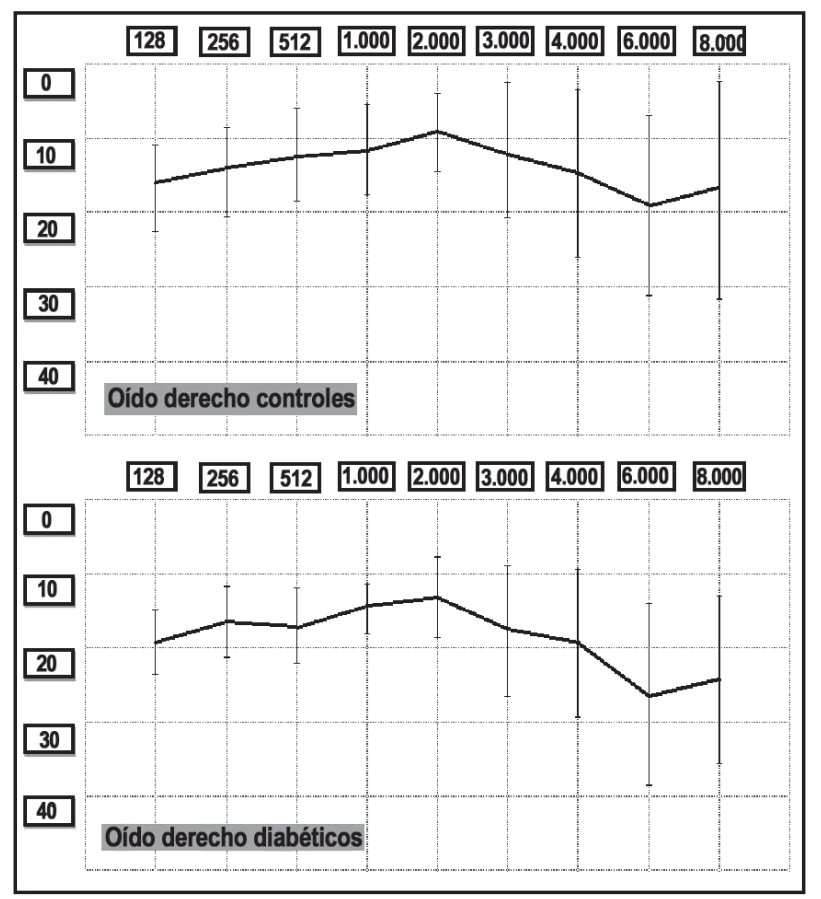

Figura 4. Umbrales auditivos oído izquierdo de pacientes controles y diabéticos con desviación típica. 


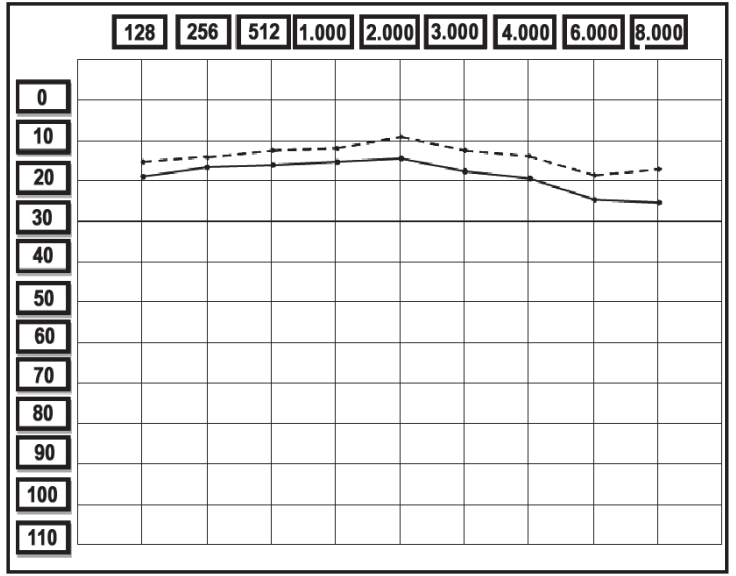

Figura 5. Promedio de umbrales auditivos derecho e izquierdo, línea continua corresponde a pacientes diabéticos y línea discontinua corresponde a pacientes controles.

Tabla 5. Media de promedio tonal puro de grupo diabéticos y controles

\begin{tabular}{|c|c|c|c|}
\hline & $\begin{array}{c}\text { Media PTP } \\
\text { diabéticos }\end{array}$ & $\begin{array}{c}\text { Media PTP } \\
\text { controles }\end{array}$ & $\mathrm{P}$ \\
\hline $\begin{array}{c}\text { Oído } \\
\text { derecho }\end{array}$ & 15,766 & 12,213 & $<0,001$ \\
\hline $\begin{array}{c}\text { Oído } \\
\text { izquierdo }\end{array}$ & 16,552 & 11,145 & $<0,001$ \\
\hline
\end{tabular}

El inicio cada vez más precoz de la obesidad infantil, la de los adolescentes y adultos jóvenes así como el número creciente de resistencia a la insulina explican el debut de la enfermedad en individuos más cada vez más jóvenes.

Es lógico suponer que si lo anterior es una realidad tendremos más prontamente las consecuencias de esta enfermedad en todos sus aspectos incluyendo al que nos interesa y que es el auditivo.

No es completamente claro que la diabetes mellitus tipo 2 favorezca una disminución de la audición. Para algunos sí se produce una hipoacusia neurosensorial $6,12,15,16,18$, mientras que otros no lo han podido demostrar ${ }^{2,14,17}$.

En nuestro trabajo se reclutaron pacientes adultos de entre 30 a 50 años de ambos sexos, en plena edad productiva y elegidos así para no tener el efecto de la pérdida auditiva por envejecimiento. Durante la entrevista en ambos grupos no hubo referencia a baja de audición lo que está de acuerdo con los criterios de exclusión planteados para ambos grupos.

Encontramos, en el grupo de los pacientes diabéticos, un deterioro de la media de los umbrales auditivos, en comparación al grupo control en casi todas las frecuencias. Estas diferencias son estadísticamente significativas $(p<0,05)$.

Como era de esperarse el promedio tonal puro también demostró lo mismo.

El análisis de los datos si bien estadísticamente es significativo, revela una pérdida auditiva menor y no detectado por quienes la sufren. Pese a lo controversial del tema y las evidentes dificultades de una metodología perfecta de estudio, nos parece preocupante el pequeño deterioro encontrado en diabéticos jóvenes. Si a lo anterior agregamos los efectos de la socioacusia, el trauma acústico y la presbiacusia el problema podría ser algo mayor.

\section{CONCLUSIÓN}

En nuestro estudio los pacientes diabéticos tipo 2 jóvenes presentaron un deterioro auditivo de grado menor, pero estadísticamente significativo, en relación a un grupo control representativo de la normalidad para esa edad.

\section{BIBLIOGRAFÍA}

1. Ministerio de Salud. Guía clínica 2010 Diabetes Mellitus tipo 2, Chile 2010.

2. P Mitchell, B Gopinath, CM McMahon et al. Relationship of type 2 diabetes to the prevalence, incidence and progression of age-related hearing Ioss. Diabetic Medicine 2009; 26(5): 483-8.

3. Encuesta Nacional de salud, Chile, tomo I (20092010) 138-51.

4. Hilary King MD, Ronald E. Aubert PhD, William H. Herman MD. Global Burden of Diabetes 19952025: prevalence, numerical estimates, and projections. Diabetes Care 1998; 21(4): 141431.

5. Susan T. Frisina, Frances Mapes, SungHee Kim, D. Robert Frisina, Robert D. Frisina. Characterization 
of hearing loss in aged type II diabetics. Hearing Research 2006; 211: 103-13.

6. Venkata Kakarlapudi, Robert Sawyer, Hinrich STAECKER. The effect of diabetes on sensorineural hearing loss. Otology \& Neurotology 2003; 24 : 382-6.

7. Donald F. Austin MD MPH, Dawn Konrad-Martin PhD, Susan Griest MPH, Garnett P. McMillan PhD, Daniel McDermott MS, Stephen Fausti $\mathrm{PHD}$. Diabetes-related changes in hearing. Laryngoscope 2009; 119(9): 1788-96.

8. Smith TL, Raynor E, Prazma J, Buenting JE, Pillsbury $\mathrm{HC}$. Insulin-dependent diabetic microangiopathy in the inner ear. Laryngoscope 1995; 105(3Pt1): 236-40.

9. Hyun-Seok Lee MD, Ki Ryung Kim Won-Ho Chung MD, Yang-Sun Cho MD, Sung Hwa Hong MD. Early sensorineural hearing loss in $\mathrm{Ob} / \mathrm{Ob}$ mouse, an animal model of type 2 Diabetes. Clinical and Experimental Otorhinolaryngology 2008; 1(4): 211-6.

10. Wackym PA, Linthicum FH JR. Diabetes Mellitus and hearing loss: clinical and histopathologic relationships. The American Journal of Otology 1986; 7(3): 176-82.

11. Fukushima H, Cureoglu S, Schachern Pa, Paparella MM, Harada T, OKTaY MF. Effects of type 2 Diabetes Mellitus on cochlear structure in humans. Archives of Otolaryngology - Head \& Neck Surgery 2006; 132(9): 934-8.
12. Cullen JR, Cinnamond MJ. Hearing loss in diabetics. The Journal of Laryngology and Otology 1993; 107: 179-82.

13. Luz Verónica Díaz de León-Morales, Kathrine Jauregui-Renaud, María Eugenia Garay-Sevilla, José Hernandez-Prado, Juan Manuel Malacara-Hernandez JM. Auditory impairment in patients with type 2 Diabetes Mellitus. Archives of Medical Research 2005; 36(5): 507-10.

14. De España R, Biurrun O, Lorente J, Traserra J. Hearing and diabetes. ORL: Journal for Oto-rhino-laryngology and its related specialties 1995; 57: 325-7.

15. Thiago Hernández Diniz, Heraldo Lorena Guida. Hearing loss in patients with Diabetes Mellitus. Brazilian Journal of Otorhinolaryngology 2009; 75(4): 573-8.

16. Dawn Konrad-Martin PhD, Donald F. Austin MD MPH, Susan Griest MPH, Garnett P. McMillan PhD, Daniel McDermott MS, Stephen Fausti PhD. Diabetes-related changes in auditory brainstem responses. Laryngoscope 2010; 120: 150-8.

17. Josef Shargorodsky MD MPH, Sharon G. Curhan MD ScM, Roland Eavey MD SM, Gary C. Curhan MD ScD. A Prospective study of cardiovascular risk factors and incident hearing loss in men. Laryngoscope 2010; 120(9): 1887-91.

18. Jianmin Ren, Peng Zhao, Li Chen, Anting Xu, Stacey N. Brown, XiaOYan XIaO. Hearing loss in middle-aged subjects with type 2 Diabetes Mellitus. Archives of Medical Research 2009; 40(1): 18-23.

Dirección: Dr. César Imarai B.

Hospital Clínico de la Universidad Católica de Chile

E-mail: caimarai@yahoo.com 